\title{
Herpes zoster and postherpetic neuralgia
}

\section{Peter N. Watson MD}

Previously published at www.cmaj.ca

$\infty \quad$ See related research article by Drolet and colleagues, page 1731

"I wish I could state anything very satisfactory as to treatment of the afterpains, which are sometimes so severe as to make the patient weary of existence." - William Bowman (1867)

$\mathrm{S}$ ome progress has been made in preventing and treating the recurrence of the chickenpox or varicella-zoster virus - known as shingles or herpes zoster - since Bowman made this statement, but there is still much to be done. Many people over 60 years of age have probably experienced herpes zoster and its consequences, because the disease is common, directly related to age, has frequent complications and can be severe, even lifelong. Drolet and colleagues have documented the burden of herpes zoster in the Canadian population by reporting an important impact on quality of life. ${ }^{2}$ The authors assessed severity of pain related to herpes zoster, effect on activities of daily living and quality of life in 261 patients with herpes zoster and the $24 \%$ in whom postherpetic neuralgia developed. They found that the disease had a major impact on sleep, enjoyment of life, general activities, mood, and levels of anxiety and depression.

Herpes zoster is the most common neurologic disorder, and postherpetic neuralgia is its most common and feared complication. In Canada each year, there are 130000 new cases of herpes zoster, 17000 cases of postherpetic neuralgia and 20 deaths. Postherpetic neuralgia can result in severe pain; patients are often unable to wear clothing that comes in contact with the lesions or to be outside in the wind because of the sensitivity of the skin on the thorax and face (common sites). There may be loss of vision or even loss of the eye as well as facial scarring. Less frequent consequences include inflammation of the spinal cord, brain and meninges, and facial paralysis. Herpes zoster and postherpetic neuralgia will probably increase as the population ages; the incidence of postherpetic neuralgia rises from $10 \%$ among people of all ages to as high as $40 \%$ among those 50 years and older. ${ }^{4}$

Three approaches are available to deal with herpes zoster and its complications. The first is to treat established postherpetic neuralgia, a neuropathic pain. General guidelines for neuropathic pain suggest starting with a tricyclic antidepressant or a gabapentinoid (gabapentin, pregabalin), followed by an opioid if necessary. ${ }^{5}$ Unfortunately, randomized controlled trials have shown only a $20 \%-40 \%$ reduction in severity of pain. ${ }^{6}$ This approach has limited success in clinical practice, where patients may be older, have concomitant diseases and be taking other drugs. Using one category of drug is often inadequate for treating neuropathic pain such as postherpetic

\section{Key points}

- Herpes zoster and postherpetic neuralgia are common, directly related to age, and the complications can be severe and lifelong.

- Several evidence-based treatments for postherpetic neuralgia have been shown to be moderately effective.

- Early and aggressive treatment of herpes zoster can reduce the severity of symptoms and possibly the incidence of postherpetic neuralgia.

- Herpes zoster vaccine appears to be underused.

neuralgia, so combinations of different drugs have been used with the hope of achieving an additive effect. ${ }^{6}$

Second, the acute pain and rash caused by herpes zoster and possibly postherpetic neuralgia ${ }^{4,8}$ may be relieved by aggressive treatment with antivirals (famciclovir, valacyclovir) within 72 hours after the onset of the rash (or even at the stage of acute, segmental, neuropathic pain [burning or shock-like] in the forehead or midthorax, which often precedes the rash). ${ }^{4}$ Tricyclic antidepressants such as amitriptyline or gabapentinoid or both can be considered and, if the pain is severe, an opioid and even a nerve block (all untested by randomized controlled clinical trials) can be used. There is evidence that the early use of antivirals will provide relief for acute pain and hasten healing of the rash, but the evidence for using antivirals to prevent postherpetic neuralgia is weak ${ }^{4}$ or absent. ${ }^{8}$ However, this approach may at least attenuate acute pain and rash, provided treatment is administered quickly.

Third, a vaccine for preventing herpes zoster and postherpetic neuralgia has been approved in Canada for people 60 years of age and older. ${ }^{9}$ This live, attenuated vaccine reduces the development of herpes zoster by $50 \%$ and postherpetic neuralgia by $67 \% ;{ }^{9}$ even if herpes zoster occurs, postherpetic neuralgia may be attenuated. The vaccine offers a substantial effect and appears to be safe. ${ }^{9}$ Yet evidence shows that it is underused; in 2008, only $7 \%$ of potential recipients were vaccinated. ${ }^{10}$ This low coverage may be due, in part, to vaccine phobia, to the cost of the vaccine (about \$150) because it is not covered by insurance, and to the logistics of using a

Dr. Watson is with the Division of Neurology, Department of Medicine, University of Toronto, Toronto, Ont.

CMAJ 2010. DOI:10.1503/cmaj.101409 
frozen vaccine (it needs to be administered within 30 minutes after reconstitution to be effective).

The future epidemiology of herpes zoster has been recently addressed. ${ }^{11}$ It is hoped that the increasing rates of varicella vaccination among children, improved awareness of the need for timely antiviral therapy and the availability of a vaccine for adults will reduce the incidence of both herpes zoster and postherpetic neuralgia. The number of people who are susceptible to herpes zoster will increase steadily as the proportion of the elderly population - and the number of immunocompromised patients (transplant recipients, patients with HIV infection and patients with cancer receiving radiation and chemotherapy) - increases.

Varicella vaccination of children may have the paradoxical effect of increasing the rate of herpes zoster in the short term, because adults from the prevaccine era will be exposed to less exogenous virus and thus will not receive the periodic boosting of immunosurveillance that keeps the disease in check. The incidence of herpes zoster could increase substantially during the next 20 to 50 years.

In conclusion, better drugs are needed for the therapy of postherpetic neuralgia. We should continue to treat herpes zoster in a timely fashion, ideally within 72 hours of rash onset or even with the occurrence of acute segmental pain. Given the evidence we have to date, increased use of the vaccine for zoster prevention is likely the best way to diminish the impact of this disease.

This commentary was solicited and has not been peer reviewed.
Competing interests: Peter Watson has given talks for Pfizer, Purdue and Merck in the past five years. He has performed a research study of tramadol with Purdue Canada and was co-investigator on a study of pregabalin funded by Pfizer. He has completed a pilot study of the civamide patch for Winston Laboratories in Chicago.

\section{REFERENCES}

1. Bowman W. Cases of zoster or unilateral confluent herpes of the ophthalmic region. Ophth Hosp Rep 1867;6:1-11.

2. Drolet M, Brisson M, Schmader KE, et al. The impact of herpes zoster and postherpetic neuralgia on health-related quality of life: a prospective study. CMAJ 2010;182:1731-6.

3. Brisson M, Pellisier JM, Camden S, et al. The potential cost-effectiveness of vaccination against herpes zoster and post-herpetic neuralgia. Hum Vaccin 2008;4:238-45.

4. Dworkin RH, Schmader KE. The epidemiology and natural history of herpes zoster and postherpetic neuralgia. In: Watson CPN, editor. Herpes zoster and postherpetic neuralgia. Amsterdam (Netherlands): Elsevier; 2001. p. 39-64.

5. Moulin DE, Clark AJ, Gilron I, et al. Pharmacological management of chronic pain. Consensus statement and guidelines from the Canadian Pain Society. Pain Res Manag 2007;12:13-22.

6. Gilron I, Max B. Combination pharmacotherapy for neuropathic pain: current evidence and future directions. Expert Rev Neurother 2005;5:823-30.

7. Watson CPN. External validity of randomized trials in neuropathic pain. In: Rothwell PM, editor. Treating individuals: from randomized trials to personalized medicine. Philadelphia (PA): Elsevier; 2007. p. 121-30.

8. Li Q, Chen N, Yang Y, et al. Antiviral treatment for preventing postherpetic neuralgia [review]. Cochrane Database Syst Rev 2009;(2):CD006866.

9. Oxman MN, Levin MD, Johnson JR, et al. A vaccine to prevent postherpetic neuralgia in older adults. $N$ Engl J Med 2005;352:2271-84.

10. Lu PJ, Euler GL, Jumaan AO, et al. Herpes zoster vaccination among adults aged 60 years or older in the United States, 2007: uptake of the first new vaccine to target seniors. Vaccine 2009;27:882-7.

11. Bennett GJ, Watson CPN. Herpes zoster and postherpetic neuralgia: past, present and future. Pain Res Manag 2009;14:275-82.

Correspondence to: Dr. C. Peter N. Watson, 1 Sir Williams Lane,

Toronto ON M9A 1T8; peter.watson@utoronto.ca

${ }^{\mathrm{Pr} A C G U P R I L}{ }^{\circledR}$ and ${ }^{\mathrm{Pr}}$ ACGURETIG ${ }^{\circledR}$ Still fighting hypertension

$\checkmark$ Powerful BP-lowering ${ }^{1}$

$\checkmark$ 24-hour BP control demonstrated with ACCUPRIL ${ }^{1}$

$\checkmark$ he flexibility of seven dosage strengths with ACCUPRIL and ACCURETIC ${ }^{1,2}$

$\checkmark$ Al dosages at a single flat price ${ }^{3 \dagger}$

† Price does not include pharmacy professional fees.

Please refer to Product Monographs for complete dosing information.

P

TAKE A FORWARD STEP

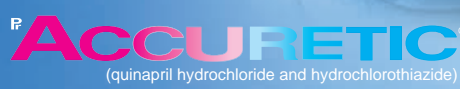

POWER OF COMBINED CONTROL
CUPRIL is indicated in essential hypertension when diuretics or betakers are unsuitable.

WARNINGS: As with all ACE inhibitors, please refer to specific warnings egarding drug discontinuation in angioedema and pregnancy.

The most frequent adverse events in controlled clinical trials with ACCUPRL ere neadache $(8.1 \%)$, dizziness $(4.1 \%)$, cough $(3.2 \%)$, fatigue $(3.2 \%)$, $2 \%$ ), nausea and/or vomiting (2.3\%) and abdominal pain $(2.0 \%)$. molete list of adverse events, please refer to the Product

in essential hypertension when combination The fixed combination is not for initial therapy.

in pregnancy. ACE inhibitors can cause fetal y and mortality when administered to pregnant use heen reported in the world literature. e used by women who are pregnant, intend to could become pregnant and who are not using measures. It is possible that quinapril passes into should be advised not to breast-feed while taking ribing information for complete contraindications. When used in pregnancy, angiotensin converting enzyme (ACE)
inhilititors can cause injury or even death of the developing fetus. When pregnancy is detected, ACCUPRIL or ACCURETIC should be discontinued as soon as possible.

The most frequent adverse events in controlled trials with ACCURETIC were headache $(6.7 \%)$, dizziness $(4.8 \%)$, cough $(3.2 \%)$ and fatigue $(2.9 \%)$. For the complete list of adverse events, please refer to the Product Monograph. 